\title{
Changes in Distribution of Lower Limb Alignment After Total Hip Arthroplasty for Crowe IV Developmental Dysplasia of the Hip
}

\author{
Jingyang Sun ${ }^{1,2, *}$ \\ Lingfei Guo ${ }^{1,2, *}$ \\ Ming $\mathrm{Ni}^{\mathrm{I}}$ \\ Junmin Shen' \\ Yinqiao Du (D) \\ Bohan Zhang ${ }^{1,2}$ \\ Guoqiang Zhang ${ }^{1,2}$ \\ Yonggang Zhou $\mathbb{D}^{1,2}$ \\ 'Department of Orthopedics, Chinese \\ People's Liberation Army General \\ Hospital, Beijing, People's Republic of \\ China; ${ }^{2}$ Medical School of Chinese \\ People's Liberation Army, Beijing, \\ People's Republic of China \\ *These authors contributed equally to \\ this work
}

Correspondence: Yonggang Zhou; Guoqiang Zhang

Department of Orthopedics, Chinese People's Liberation Army General

Hospital, Fuxing Road, Haidian District, Beijing, People's Republic of China

Tel +86I380I287599; +86I360I2I 0743

Email ygzhou30I@I63.com;

gqz301@126.com
Purpose: The purpose of this study was to evaluate the distribution of lower limb alignment in Crowe IV developmental dysplasia of the hip (DDH) before and after total hip arthroplasty (THA).

Patients and Methods: We retrospectively included 64 Crowe IV DDH patients (87 hips) who underwent THA between February 2010 and May 2019. Radiographic parameters were measured on full limb length standing anteroposterior radiographs, including hip-knee-ankle angle (HKA), mechanical lateral distal femoral angle (mLDFA), mechanical medial proximal tibial angle (mMPTA), anatomical lateral distal femoral angle (aLDFA), and anatomical tibiofemoral angle (aTFA).

Results: HKA improved from $176.54^{\circ} \pm 3.52^{\circ}$ preoperatively to $179.45^{\circ} \pm 4.31^{\circ}$ at the last followup ( $\mathrm{P}<0.001)$. According to the preoperative HKA, 40 hips were defined as knee valgus alignment. The majority of them were characteristic of a valgus mLDFA and a valgus or neutral mMPTA $(35 \%, 47.5 \%)$. After THA, there were still 22 hips defined as knee valgus alignment. More than $50 \%$ of them were characteristic of a valgus mLDFA and a neutral mMPTA. Five hips $(22.7 \%)$ revealed valgus alignment in both mLDFA and mMPTA. Twenty-one hips $(24.1 \%)$ remained knee valgus alignment before and after surgery. One hip, defined as neutral alignment, preoperatively turned into valgus alignment after surgery. Conclusion: Both preoperative and postoperative sources of valgus alignment were mainly from the femoral side among Crowe IV DDH patients. THA mainly played a positive role in the reconstruction of lower limb alignment in these patients.

Keywords: total hip arthroplasty, Crowe IV, developmental dysplasia of the hip, knee alignment

\section{Introduction}

Total hip arthroplasty (THA) is an efficient treatment option for adult patients with osteoarthritis secondary to Crowe IV developmental dysplasia of the hip (DDH). ${ }^{1}$ Favorable results have been reported in many studies regarding these complicated cases after THA. ${ }^{2-5}$ However, there remained some patients complaining about the knee valgus deformity. ${ }^{6}$ Compared with limb length discrepancy (LLD) after THA, knee valgus deformity was less concerned by researchers. The emerging role of knee valgus deformity might potentially result in patient's dissatisfaction after THA.

It should be noted that knee valgus alignment naturally exists in some of these patients before surgery. Developmental changes have been observed in their knees on 
the ipsilateral side, such as hypoplasia of lateral femoral condyle and relative valgus tibial plateau, which all contribute to overall knee valgus alignment. ${ }^{7-9}$ Apart from the anatomical abnormalities of native knee, limb lengthening after THA was also supposed to be a reason for deterioration of knee valgus alignment. Kilicarslan et al prospectively evaluated 30 hips of 22 Crowe III/IV patients who had normal knees. In the early postoperative period, genu valgum was observed in all knees on the operated side. The Q angle increased by an average of $4.4^{\circ}$. They attributed those pathological changes to the strain in the iliotibial tract, which resulted from limb lengthening after reducing the hip center to the anatomical position. ${ }^{6,10}$

On the contrary, there were also some studies showing the positive effect of THA on coronal alignment of the lower limb in those patients. Kocabiyik et al reviewed the full-length lower limb radiographs of 25 Crowe IV patients. At the 1-year after THA, they found that modification of femoral offset and reconstruction of hip joint anatomy led to neutralization of knee valgus alignment. ${ }^{11}$ Moreover, Zhao et al evaluated the coronal alignment of the lower limb in 50 patients with Crowe IV DDH before surgery, immediately after surgery, and two years later. By implantation of the acetabular cup at the level of anatomic hip center, they found that coronal alignment of the operated limb was immediately altered, and valgus inclination was significantly reduced. And the knee alignment did not change significantly after 2-year followup. ${ }^{12}$ However, we supposed that the actual alignment of some patients might be masked by the overall improvement of alignmentrelated radiographic parameters. Until now, there have been no studies focusing on the distribution of the coronal alignment of the lower limb in these patients, both preoperatively and postoperatively.

Therefore, the purpose of this study was to evaluate the distribution of knee alignment in Crowe IV DDH patients both preoperatively and postoperatively. We also tried to answer the following questions: 1) what is the main source of knee valgus alignment before and after THA, femoral side, tibial side or both? 2) would THA have a positive or negative effect on the knee alignment?

\section{Patients and Methods}

\section{Patients}

After the approval of our institutional review board, we retrospectively reviewed the Crowe IV DDH patients who underwent THA between February 2010 and May 2019 by one senior surgeon in our hospital. The inclusion criteria were as follows: patients with Crowe IV DDH on at least one side; patients who had a full limb length standing anteroposterior (AP) radiograph both preoperatively and at least 1 year postoperatively. Exclusion criteria contained: patients with angulated extra-articular deformities on the femoral or tibial side; patients with radiographic evidence of cartilage abrasion in either knee; nonstandard radiographs which cannot be measured due to improper flexion or rotation of the knee. Finally, a total of 64 patients ( 87 hips) were included in the study. Demographics and clinical information including age, gender, weight, height, and surgical manipulations were collected from our electronic medical records.

\section{Surgical Procedure}

All operations were performed by one senior surgeon under general anesthesia in the lateral decubitus position through a posterolateral approach. The detailed surgical procedure was almost the same as previously described in the literatures. ${ }^{13,14}$ All acetabular cups were implanted at the anatomical position. No bone grafts were used to increase the cup coverage. On the femoral side, an identical modular femoral stem (S-ROM, DePuy, Warsaw, Indiana) was used. After preparation of the femoral canal, we tried to reduce the femoral head with constant and vigorous traction. If hip reduction cannot be done, the distance between the cup center and femoral head center was recorded, and the decision on femoral shortening was made. We performed a subtrochanteric osteotomy at the site of approximately $1-2 \mathrm{~cm}$ beneath the lesser trochanter by resection of a length of femur based on the distance we recorded before, leaving a scope of $1-1.5 \mathrm{~cm}$ with surgeon's discretion. Soft tissue release included joint capsulectomy, gluteal sling release, iliopsoas tenotomy, and percutaneous partial adductor tenotomy if needed. Impingement-free range of motion was tested in three positions: $90^{\circ}$ hip flexion, $45^{\circ}$ hip internal rotation, and excessive hip extension. LLD was assessed with reference to the inferior point of bilateral patella. Mild LLD and joint laxity could be compensated by means of the modifications of head/neck length, offset, and stem depth in femur. Postoperatively, patient's hip and knee were maintained in flexion for several days to relax the sciatic nerve and reduce tension of soft tissue.

\section{Radiographic Measurement}

All full limb length standing AP radiographs were obtained using a standard protocol using GE Definium 6000 digital radiography (DR) system (GE Healthcare, USA). ${ }^{15}$ The 
requirements for standing posture during filming were as follows: stand facing the X-ray tube with the tibial tubercle pointing anteriorly; keep their legs straight and full weightbearing; allowed tiptoeing if needed but not to place wooden blocks under the short limb to make pelvis level. The radiographs were viewed and measured on the PACS software in hospital (Medcare, Qingdao, China).

There were five radiographic parameters measured in each affected limb before surgery and/or after surgery, including hip-knee-ankle angle (HKA), mechanical lateral distal femoral angle (mLDFA), mechanical medial proximal tibial angle (mMPTA), anatomical lateral distal femoral angle (aLDFA), and anatomical tibiofemoral angle (aTFA) (Figure 1). The measurement methods of these radiographic parameters were by reference to previous studies. ${ }^{9,16,17} \mathrm{HKA}$ is defined as the lateral angle between the mechanical axis of the femur and the tibia. mLDFA is defined as the lateral angle between the joint line of the distal femur and the femoral mechanical axis. MMPTA is defined as the medial angle between the joint line of the tibial plateau and the tibial mechanical axis. aLDFA is defined as the lateral angle between the joint line of distal femur and the femoral anatomical axis. aTFA is defined as the lateral angle between the anatomical axis of femur and tibia. The scales of these parameters (applied for defining valgus, neutral, and varus alignment) were based on the values used in previous literatures and shown in Table 1. ${ }^{9,17}$ Finally, we defined the knee alignment according to the HKA angle.

In order to assess the inter-observer variability and intraobserver reliability, measurement was performed twice by two trained arthroplasty surgeons independently in random order with an interval of at least 1 month. Before initiation of the measurement, two observers reached an agreement on criteria for radiographic measurement and all identifying information was removed. Assessment of inter- and intraobserver consistency was determined by the intra-class correlation coefficient (ICC). Agreement was graded as slight (0 to 0.2 ), fair ( 0.21 to 0.40$)$, moderate ( 0.41 to 0.60$)$, substantial $(0.61$ to 0.80$)$ or almost perfect $(0.81$ to 1.0$){ }^{18}$

\section{Statistical Analysis}

Categorical variables were presented as frequencies, and continuous variables as means and standard deviation. Categorical variables were compared using Chi-square test. Paired $t$ test was used to compare the preoperative and postoperative radiographic parameters. All statistical analyses were performed using SPSS version 26.0 (IBM Inc., Armonk, New York). $\mathrm{P}<0.05$ was considered statistically significant.

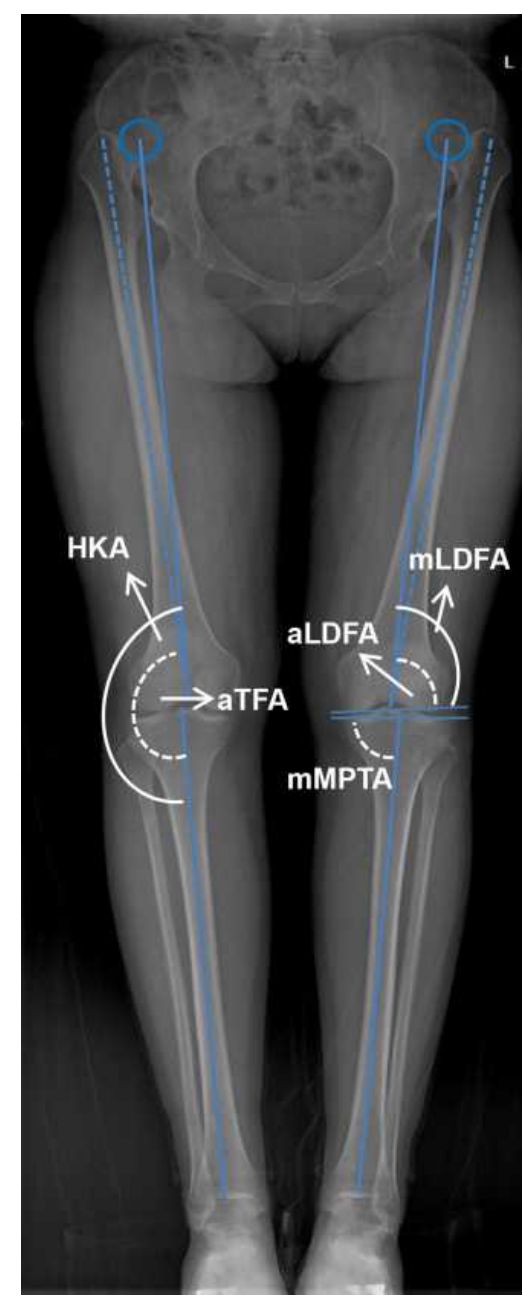

Figure I The diagram shows the methods of radiographic measurement. Abbreviations: HKA, hip-knee-ankle angle; aTFA, anatomical tibiofemoral angle; mLDFA, mechanical lateral distal femoral angle; mMPTA, mechanical medial proximal tibial angle; aLDFA, anatomical lateral distal femoral angle.

\section{Results}

Demographic and clinical data are shown in Table 2. The results of radiographic measurements showed nearly perfect reliability of intra- and inter-observer agreements (ICC > 0.81). Overall, HKA improved from $176.54^{\circ}$

Table I The Scales of Radiographic Parameters Applied for Defining Valgus, Neutral, and Varus Alignment

\begin{tabular}{|l|l|l|l|}
\hline Parameter & Valgus & Neutral & Varus \\
\hline HKA & $<177.0^{\circ}$ & $177.0^{\circ}-183.0^{\circ}$ & $>183.0^{\circ}$ \\
mLDFA & $<85.0^{\circ}$ & $85.0^{\circ}-90.0^{\circ}$ & $>90.0^{\circ}$ \\
mMPTA & $>90.0^{\circ}$ & $85.0^{\circ}-90.0^{\circ}$ & $<85.0^{\circ}$ \\
aLDFA & $<79.0^{\circ}$ & $79.0^{\circ}-83.0^{\circ}$ & $>83.0^{\circ}$ \\
aTFA & $<170.0^{\circ}$ & $170.0^{\circ}-175.0^{\circ}$ & $>175.0^{\circ}$ \\
\hline
\end{tabular}

Abbreviations: HKA, hip-knee-ankle angle; mLDFA, mechanical lateral distal femoral angle; mMPTA, mechanical medial proximal tibial angle; aLDFA, anatomical lateral distal femoral angle; aTFA, anatomical tibiofemoral angle. 
Table 2 Demographic and Clinical Data of Patients

\begin{tabular}{|l|l|}
\hline Characteristic & Value \\
\hline Sex, $\mathrm{n}(\%)$ & \\
Male & $4(6)$ \\
Female & $60(94)$ \\
Age (years) (mean \pm SD) & $40.03 \pm \mathrm{II} .2 \mathrm{I}$ \\
Height $(\mathrm{m})($ mean $\pm \mathrm{SD})$ & $1.59 \pm 0.12$ \\
Weight $(\mathrm{kg})($ mean $\pm \mathrm{SD})$ & $55.09 \pm 9.45$ \\
BMl $\left(\mathrm{kg} / \mathrm{m}^{2}\right)($ mean $\pm \mathrm{SD})$ & $21.94 \pm 3.49$ \\
\hline Side, $\mathrm{n}(\%)$ & \\
Unilateral & $4 \mathrm{I}(64)$ \\
Bilateral & $23(36)$ \\
\hline Subtrochanteric shortening osteotomy, $\mathrm{n}(\%)$ & \\
Yes & $6 \mathrm{I}(70)$ \\
No & $26(30)$ \\
Followup duration (months) (range) & $12-108$ \\
\hline
\end{tabular}

Abbreviations: BMI, body mass index; SD, standard deviation.

$\pm 3.52^{\circ}$ preoperatively to $179.45^{\circ} \pm 4.31^{\circ}$ at the last followup ( $\mathrm{P}<0.001)$. Considering solely the femoral side, mLDFA increased from $83.25^{\circ} \pm 3.08^{\circ}$ to $85.89^{\circ} \pm 3.66^{\circ}$ $(\mathrm{P}<0.001)$. From the perspective of aTFA, after surgery, its value increased from $170.87^{\circ} \pm 14.64^{\circ}$ to $174.82^{\circ} \pm 4.27^{\circ}$ $(\mathrm{P}<0.001)$.

The distribution of radiographic parameters before surgery is shown in Figure 2. Obviously, mLDFA and aLDFA of most limbs are defined as valgus alignment (48.3\%, $63.2 \%$ ). Besides, $32.2 \%$ of mMPTA are defined as valgus alignment. After THA, the distribution of radiographic parameters related to surgery changed and is shown in Figure 3. It could be seen that the proportion of radiographic parameters defined as valgus alignment decreased, and meanwhile the proportion of the ones defined as neutral and varus alignment increased.

According to the preoperative HKA, 40 hips were defined as knee valgus alignment. The source of preoperative valgus alignment among these 40 hips is displayed in Table 3. The majority of them were characteristic of a valgus MLDFA and a valgus or neutral mMPTA (35\%, 47.5\%). After THA, there were still 22 hips defined as knee valgus alignment. The source of postoperative valgus alignment among these 22 hips is displayed in Table 4 . More than $50 \%$ of them were characteristic of a valgus mLDFA and a neutral mMPTA. Five hips (22.7\%) revealed valgus alignment in both mLDFA and mMPTA.

After THA, the transition of knee alignment is shown in Table 5 and Figure 4. Twenty-one hips (24.1\%) remained knee valgus alignment before and after surgery. One hip, defined as neutral alignment, preoperatively turned into valgus alignment after surgery. Besides, 17 hips (19.6\%) with knee valgus alignment were corrected for neutral alignment (Figure 5) and even 2 hips (2.3\%) to varus alignment. After THA, 50 hips were defined as neutral alignment. Interestingly, 15 hips $(17.2 \%)$ were defined as knee varus alignment postoperatively, which came from 2 valgus alignment, 12 neutral alignment, and 1 varus alignment before surgery (Figure 6).

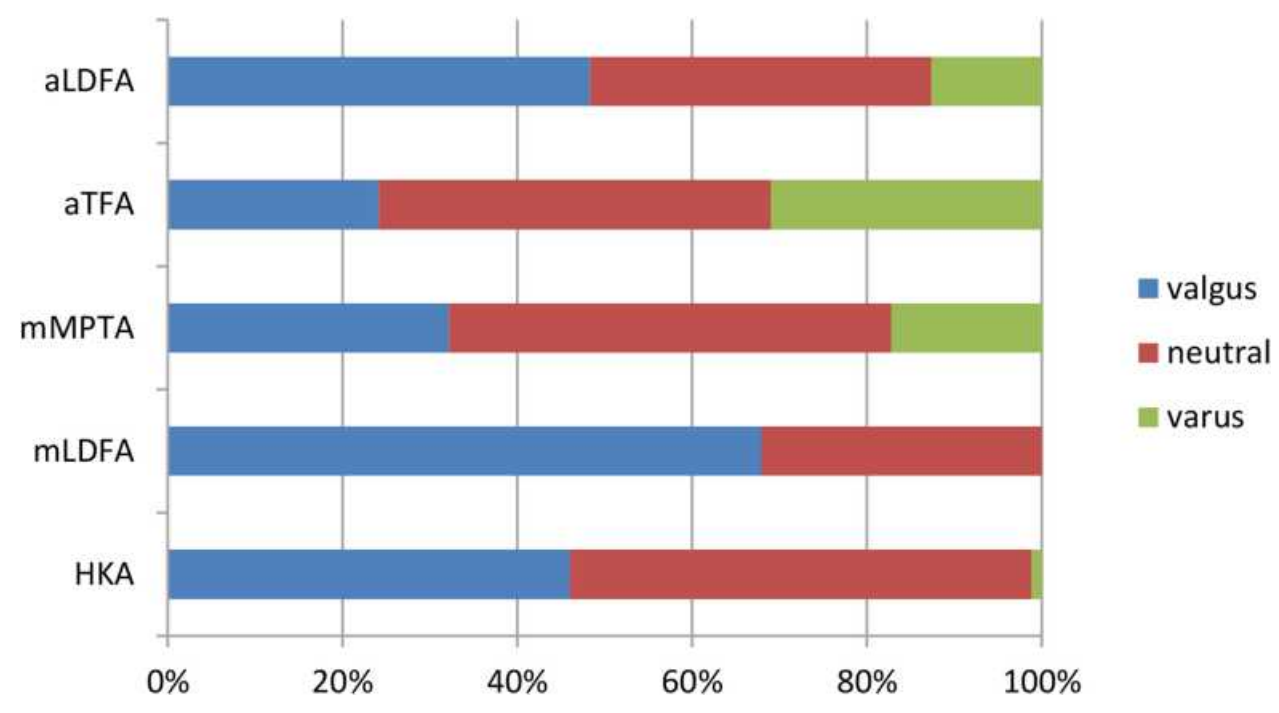

Figure 2 The distribution of preoperative radiographic parameters which are related to lower limb alignment.

Abbreviations: HKA, hip-knee-ankle angle; mLDFA, mechanical lateral distal femoral angle; mMPTA, mechanical medial proximal tibial angle; aTFA, anatomical tibiofemoral angle; aLDFA, anatomical lateral distal femoral angle. 


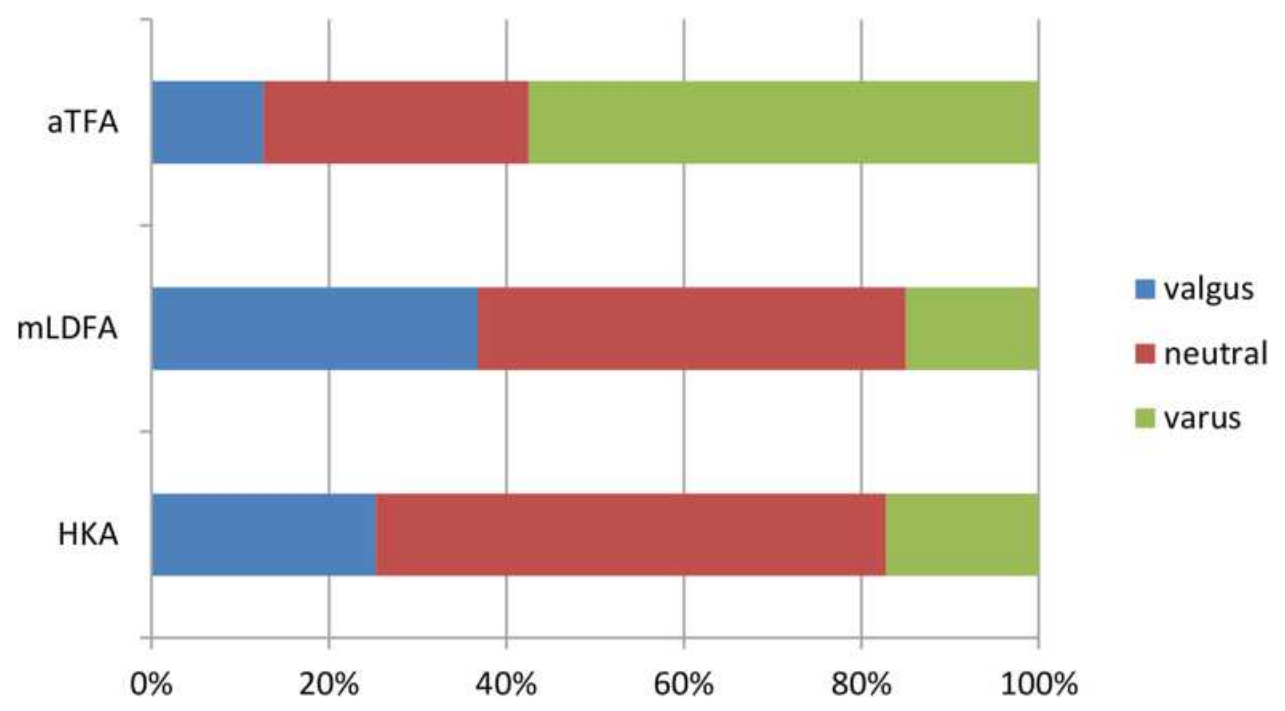

Figure 3 The distribution of postoperative radiographic parameters which are related to lower limb alignment.

Abbreviations: HKA, hip-knee-ankle angle; mLDFA, mechanical lateral distal femoral angle; aTFA, anatomical tibiofemoral angle.

\section{Discussion}

In recent years, the knee valgus alignment among Crowe IV DDH patients either before surgery or after surgery has been given more and more attention. ${ }^{9,11,12,16,19}$ The valgus alignment of the lower limb may cause knee pain, accelerated degeneration in the lateral

Table 3 The Source of Valgus Alignment Among the Limbs Which Was Defined as Knee Valgus Before Surgery

\begin{tabular}{|l|l|l|l|l|}
\hline \multirow{2}{*}{ mMPTA $\dagger$} & \multicolumn{3}{|c|}{ mLDFA $^{\dagger}$} & \multirow{2}{*}{ Total } \\
\cline { 2 - 4 } & Valgus & Neutral & Varus & \\
\hline Valgus & $14(35)$ & $3(7.5)$ & 0 & $17(42.5)$ \\
Neutral & $19(47.5)$ & $\mathrm{I}(2.5)$ & 0 & $20(50)$ \\
Varus & $2(5)$ & $\mathrm{I}(2.5)$ & 0 & $3(7.5)$ \\
Total & $35(87.5)$ & $5(12.5)$ & 0 & 40 \\
\hline
\end{tabular}

Note: ${ }^{\dagger}$ Data were given as the number of hips with the percentage in parentheses. Abbreviations: mLDFA, mechanical lateral distal femoral angle; mMPTA, mechanical medial proximal tibial angle.

Table 4 The Source of Valgus Alignment Among the Limbs Which Was Defined as Knee Valgus After Surgery

\begin{tabular}{|l|l|l|l|l|}
\hline \multirow{2}{*}{ mMPTA $^{\dagger}$} & \multicolumn{3}{|c|}{ mLDFA $^{\dagger}$} & \multirow{2}{*}{ Total } \\
\cline { 2 - 4 } & Valgus & Neutral & Varus & \\
\hline Valgus & $5(22.7)$ & $3(13.7)$ & 0 & $8(36.4)$ \\
Neutral & $12(54.6)$ & $1(4.5)$ & 0 & $13(59.1)$ \\
Varus & $1(4.5)$ & 0 & 0 & $1(4.5)$ \\
Total & $18(81.8)$ & $4(18.2)$ & 0 & 22 \\
\hline
\end{tabular}

Note: ${ }^{\dagger}$ Data were given as the number of hips with the percentage in parentheses. Abbreviations: mLDFA, mechanical lateral distal femoral angle; mMPTA, mechanical medial proximal tibial angle. compartment, soft tissue imbalance, and abnormal gait, all of which contribute to the postoperative dissatisfaction among these patients. ${ }^{6,20}$ In this study, a detailed profile of knee alignment among Crowe IV DDH patients before and after THA was presented. Overall, an evident improvement in knee alignment was observed after THA, either in the aspect of actual distribution or considering the mean values of radiographic parameters. Furthermore, based on the transition of alignment after surgery, it seems that THA mainly plays a positive role in the reconstruction of lower limb alignment among Crowe IV DDH patients. Both preoperative and postoperative sources of valgus alignment were mainly from the femoral side. These findings revealed clinical significance for arthroplasty surgeons to make surgical plans and to set reasonable expectations for Crowe IV DDH patients.

Table 5 HKA Value of Each Group of Lower Limb Alignment Before and After Surgery

\begin{tabular}{|l|l|l|}
\hline \multirow{2}{*}{ Group } & \multicolumn{2}{|c|}{ HKA (Mean士SD, Range) } \\
\cline { 2 - 3 } & Before Surgery & After Surgery \\
\hline \multirow{2}{*}{ Valgus } & $172.70^{\circ} \pm 2.22^{\circ}\left(166.42^{\circ}-\right.$ & $174.01^{\circ} \pm 2.83^{\circ}\left(168.81^{\circ}-\right.$ \\
& $\left.174.52^{\circ}\right)$ & $176.87)$ \\
Neutral & $178.87^{\circ} \pm 1.38^{\circ}\left(177.29^{\circ}-\right.$ & $179.56^{\circ} \pm 1.80^{\circ}\left(177.05^{\circ}-\right.$ \\
& $\left.182.85^{\circ}\right)$ & $\left.182.44^{\circ}\right)$ \\
Varus & $183.44^{\circ}$ & $186.02^{\circ} \pm 2.41^{\circ}\left(183.53^{\circ}-\right.$ \\
& & $\left.191.96^{\circ}\right)$ \\
\hline
\end{tabular}

Abbreviations: HKA, hip-knee-ankle angle; SD, standard deviation. 


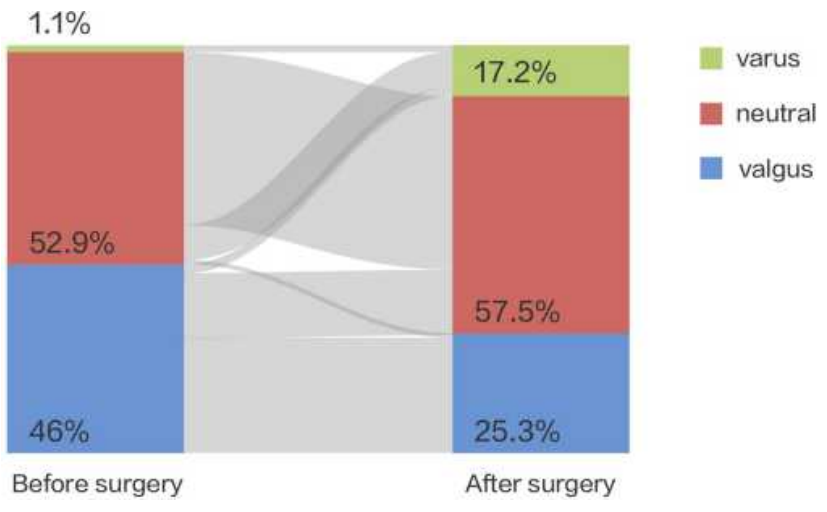

Figure 4 A Sankey diagram showing the transitions of lower limb alignment before and after surgery. The width of grey line visually represented the number of limbs in different transitions.

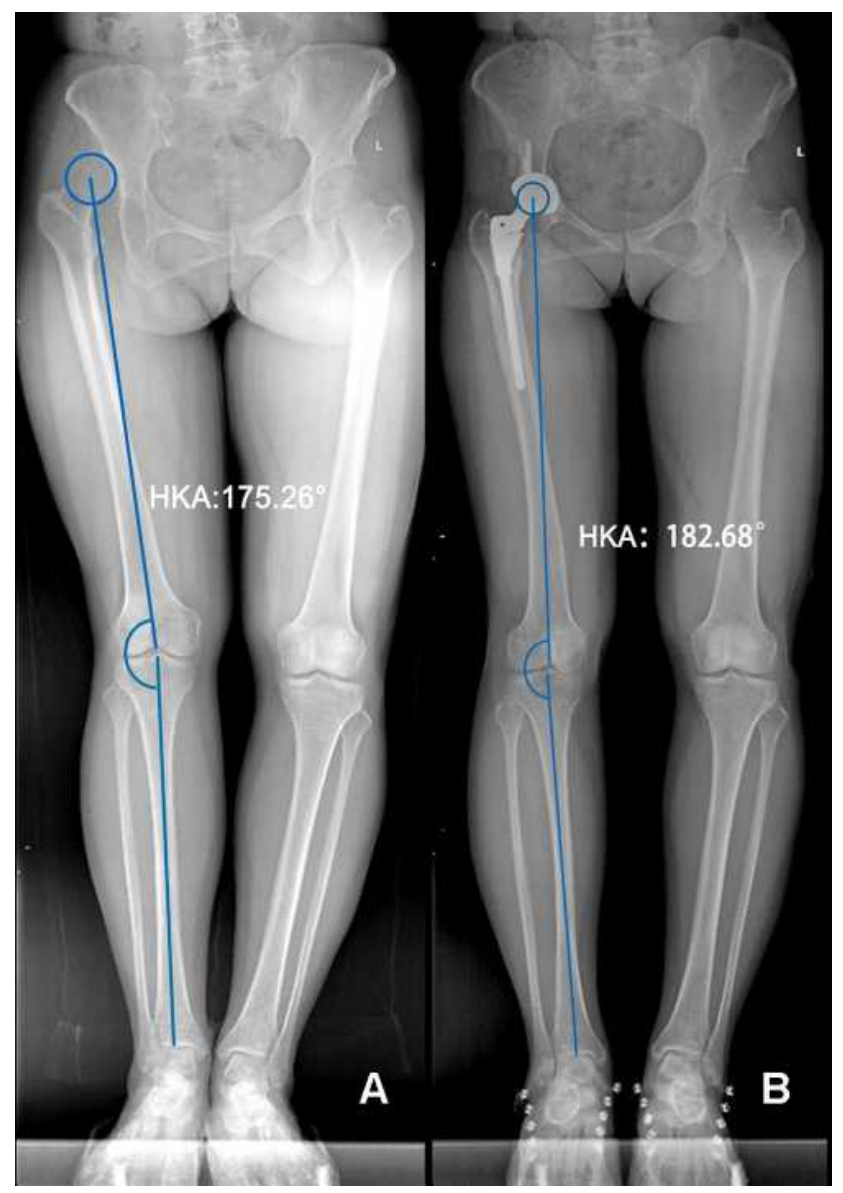

Figure 5 The full limb length $X$-ray shows that a 43-year-old woman have a transition of lower limb alignment from valgus to neutral after THA. (A) Preoperative $\mathrm{X}$-ray. (B) Postoperative $\mathrm{X}$-ray.

As for the first question, it could be seen that the main source of knee valgus alignment was from the femoral side. This conclusion was in agreement with that of Zhang et al. ${ }^{12}$ According to the morphological

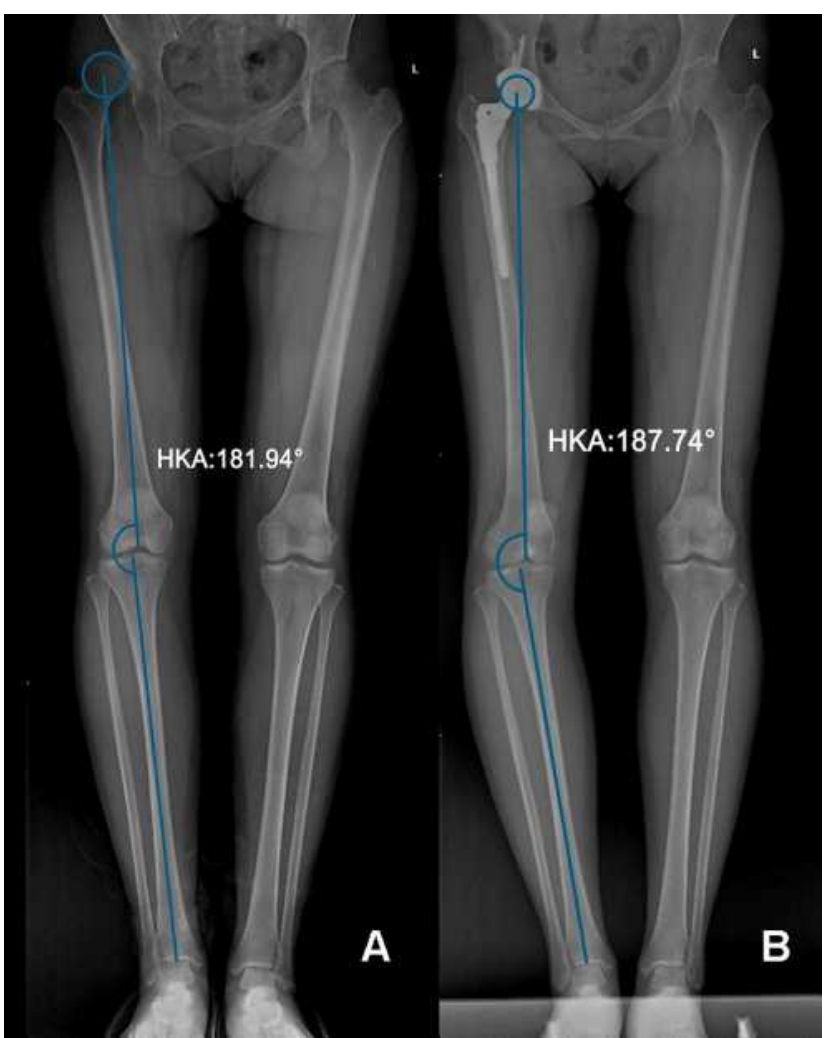

Figure 6 The full limb length X-ray shows that a 35-year-old woman have a transition of lower limb alignment from neutral to varus after THA. (A) Preoperative X-ray. (B) Postoperative X-ray.

study of knee joint among Crowe IV DDH patients, the medial femoral condyle generally becomes larger vertically, while the lateral condyle remains relatively small. ${ }^{7,8}$ Apart from the commonly greater lateral inclination of the femur, a tibial plateau was also found in valgus among some patients. ${ }^{7,9}$ However, in our study, knee valgus alignment solely resulting from greater mMPTA was uncommon both before and after surgery. More often, the valgus tibial plateau exerted an auxiliary effect on knee valgus alignment. Therefore, the reconstruction of mLDFA is especially important. Benefitting from the modularity of the femoral stem used in this study, we were adequate to adjust the femoral anteversion and femoral offset, which optimized the limb alignment on coronal plane. With respect to those cases with especially small mLDFA, a subsequent distal femoral varus osteotomy may be an inevitable option for them. ${ }^{21,22}$

On the second question, results of current literatures were more controversial. Represented by Kilicarslan et al, they supposed that genu valgum developed among Crowe III/IV DDH patients due to limb lengthening, which was 
unavoidable when implanting the cup component at the anatomical position. ${ }^{6}$ On the other, both the results of Kocabiyik et al and Zhao et al showed a significant improvement in HKA angle after surgery for Crowe IV DDH patients, indicating the positive role of THA. ${ }^{11,12}$ However, they drew the conclusion based on the overall HKA angle, not the actual distribution of knee alignment. The results of our study just made up for their shortcomings. According to the distribution of HKA, we observed nearly a half reduction of knee valgus alignment after THA. We further assessed the change in knee alignment distribution before and after surgery (Figure 4). Approximately half of those hips defined as valgus alignment preoperatively turned into neutral or varus alignment after THA. Only 1 hip defined as non-valgus alignment preoperatively turned into valgus alignment after THA. This indirectly reflected the positive effect of THA on knee alignment in Crowe IV DDH patients. It is worth noting that our results could not rule out the possibility of genu valgum development due to limb lengthening in the early postoperative period. ${ }^{20}$ Different from the results of Kilicarslan et al, we only found 1 normal knee developing genu valgum, not like their total 30 knees. ${ }^{6}$ We supposed that the longer followup time ( $\geq 1$ year) could partly explain the enormous difference. The strain on the iliotibial tract might gradually diminish with the progression of rehabilitation exercise. ${ }^{23,24}$

In addition to the improvement of knee alignment benefitted by THA, we should also concerned about the remaining non-neutral knee alignment after surgery. As previously mentioned, nearly half of valgus knees preoperatively remained unchanged after THA, with the postoperative HKA angle ranged from $168.81^{\circ}$ to $176.87^{\circ}$. Patients who complained of knee pain in the lateral compartment and had limping gait due to the genu valgum may be indicated for distal femoral varus osteotomy. ${ }^{21}$ Moreover, beyond our expectation, the proportion of varus alignment increased from $1.1 \%$ preoperatively to $17.2 \%$ postoperatively. However, only $5 / 15$ of these hips had a varus mLDFA after surgery. Similar to the theory of "long leg arthropathy" and "windswept deformity" which were used to describe the alignment of contralateral knee by Someya et al ${ }^{19}$ we speculated that knee varus alignment after surgery could be caused by soft tissue imbalance secondary to LLD and/or pelvic obliquity. Further study should be performed to explore the contributing factor to abnormal knee alignment.

Our study has some limitations. First, this is a retrospective study with its intrinsic bias. However, the data we acquired were mainly from objective radiographs, with no recall bias. Secondly, our cohort is not a consecutive case series due to data integrity, and therefore selection bias may not be avoidable. However, our study appears to be the largest study so far focusing on the distribution of knee alignment among these patients both before and after THA. Thirdly, the followup time is variable. However, we suppose that a minimum of one-year followup would warrant a constant knee alignment. Furthermore, a longitudinal and sequential observation of the knee alignment may be more meaningful. Fourthly, due to the possible effects of other factors including LLD, pelvic obliquity, and femoral offset, a multivariate analysis would be needed to further elucidate the results in this study. Fifthly, physical examination and clinical scoring would be more useful for a comprehensive evaluation of knee joint.

\section{Conclusion}

Both preoperative and postoperative sources of valgus alignment were mainly from the femoral side among Crowe IV DDH patients. Knee alignment of the operated limb gained significant improvement after THA. Based on the transition of alignment before and after surgery, THA played a positive role in the reconstruction of lower limb alignment in these patients.

\section{Ethical Approval}

This retrospective review study involving human participants was in accordance with the ethical standards of the institutional and national research committee and with the 1964 Helsinki Declaration and its later amendments or comparable ethical standards. This study was approved by the medical ethics committee of Chinese PLA General Hospital (S2020-138-01).

\section{Informed Consent}

Informed written consents to participate in the research and to publish the details were obtained from all individual participants prior to the commencement of the study.

\section{Acknowledgments}

The authors would like to thank all staff from the participating departments and clinics. Specifically, we would like to thank the medical illustrator (Xinghui Li) for painting of Sankey diagram. Jingyang Sun and Lingfei Guo are considered co-first authors. 


\section{Funding}

This research did not receive any specific grant from funding agencies in the public, commercial, or not-forprofit sectors.

\section{Disclosure}

The authors declare that they have no competing interests.

\section{References}

1. Rogers B, Garbedian S, Kuchinad R, et al. Total hip arthroplasty for adult hip dysplasia. J Bone Joint Surg Am. 2012;94(19):1809-1821. doi:10.2106/JBJS.K.00779

2. Sun C, Zhang Y, Li L, et al. Long-term outcomes of total hip arthroplasty with transverse subtrochanteric shortening osteotomy and modular stem in Crowe IV developmental dysplasia. J Arthroplasty. 2020;21. doi:10.1016/j.arth.2020.08.031

3. Wang S, Zhou Y, Ma H, et al. Mid-term results of total hip replacement with subtrochanteric osteotomy, modular stem, and ceramic surface in Crowe IV hip dysplasia. Arthroplast Today. 2018;4:363-369. doi:10.1016/j.artd.2017.07.003

4. Grappiolo G, La Camera F, Della Rocca A, et al. Total hip arthroplasty with a monoblock conical stem and subtrochanteric transverse shortening osteotomy in Crowe type IV dysplastic hips. Int Orthop. 2019;43:77-83. doi:10.1007/s00264-018-4122-5

5. Wang D, Li L, Wang H, et al. Long-term results of cementless total hip arthroplasty with subtrochanteric shortening osteotomy in Crowe type IV developmental dysplasia. $J$ Arthroplasty. 2017;32:1211-1219. doi:10.1016/j.arth.2016.11.005

6. Kilicarslan K, Yalcin N, Cicek H, et al. What happens at the adjacent knee joint after total hip arthroplasty of Crowe type III and IV dysplastic hips? J Arthroplasty. 2012;27:266-270. doi:10.1016/j. arth.2011.04.014

7. Kandemir U, Yazici M, Alpaslan A, et al. Morphology of the knee in adult patients with neglected developmental dysplasia of the hip. J Bone Joint Surg Am. 2002;84:2249-2257. doi:10.2106/00004623200212000-00019

8. Li Q, Kadhim M, Zhang L, et al. Knee joint changes in patients with neglected developmental hip dysplasia: a prospective case-control study. Knee. 2014;21:1072-1076. doi:10.1016/j.knee.2014.08.012

9. Zhang Z, Zhang H, Luo D, et al. Coronal plane alignment of the lower limbs in patients with unilateral developmental hip dislocation. Int Orthop. 2018;42(12):2761-2769. doi:10.1007/s00264-018-3813-2

10. Kong X, Chai W, Chen J, et al. Intraoperative monitoring of the femoral and sciatic nerves in total hip arthroplasty with high-riding developmental dysplasia. Bone Joint J. 2019;11:1438-1446. doi:10.1302/0301-620X.101B11.BJJ-2019-0341.R2
11. Kocabiyik A, Misir A, Kizkapan T, et al. Changes in hip, knee, and ankle coronal alignments after total hip arthroplasty with transverse femoral shortening osteotomy for unilateral Crowe type IV developmental dysplasia of the hip. $J$ Arthroplasty. 2017;32(11):3449-3456. doi:10.1016/j.arth.2017.05.044

12. Zhao H, Kang P, Shi X, et al. Effects of total hip arthroplasty on axial alignment of the lower limb in patients with unilateral developmental hip dysplasia (Crowe type IV). $J$ Arthroplasty. 2019;34 (10):2406-2414. doi:10.1016/j.arth.2019.04.028

13. Zhou Y, Sun C, Wang Y. New method addressing the problem of using ceramic-on-ceramic bearing in too small acetabulum of high-riding DDH patients with THA. Semin Arthroplasty. 2012;23 (4):226-231. doi:10.1053/j.sart.2012.12.006

14. Sun J, Zhang G, Shen J, et al. Dislocation height performs well in predicting the use of subtrochanteric osteotomy in Crowe type IV hips. Ther Clin Risk Manag. 2020;16:989-997. doi:10.2147/TCRM. S272771

15. Sabharwal S, Zhao C. Assessment of lower limb alignment: supine fluoroscopy compared with a standing full-length radiograph. $J$ Bone Joint Surg Am. 2008;90:43-51. doi:10.2106/JBJS.F.01514

16. Guo S, Zhou Y, Yang D, et al. Lower-limb valgus deformity associated with developmental hip dysplasia. Chin Med $J$ (Engl). 2012;125(22):3956-3960.

17. Paley D. Principles of Deformity Correction. New York: Springer; 2002.

18. Landis J, Koch G. The measurement of observer agreement for categorical data. Biometrics. 1977;33:159-174. doi:10.2307/2529310

19. Someya S, Sonohata M, Ide S, et al. Lower limbs alignment in patients with a unilateral completely dislocated hip. Open Orthop J. 2016;10:448-456. doi:10.2174/1874325001610010448

20. Tokuhara Y, Kadoya Y, Kim M, et al. Anterior knee pain after total hip arthroplasty in developmental dysplasia. $J$ Arthroplasty. 2011;26:955-960. doi:10.1016/j.arth.2010.07.007

21. Duethman N, Bernard C, Camp C, et al. Medial closing wedge distal femoral osteotomy. Clin Sports Med. 2019;38:361-373. doi:10.1016/ j.csm.2019.02.005

22. Koulouvaris P, Stafylas K, Sculco T, et al. Distal femoral shortening in total hip arthroplasty for complex primary hip reconstruction. A new surgical technique. J Arthroplasty. 2008;23:992-998. doi:10.1016/j.arth.2007.09.013

23. Sobiech M, Jabłoński M, Gorzelak M, et al. Postoperative limb lengthening following total hip arthroplasty (THA) through a posterior approach-a challenge for the orthopaedist or physiotherapist? Ortop Traumatol Rehabil. 2010;12:420-429.

24. Lindsey C, Makarov M, Shoemaker S, et al. The effect of the amount of limb lengthening on skeletal muscle. Clin Orthop Relat Res. 2002;402:278-287. doi:10.1097/00003086-200209000-00028
Therapeutics and Clinical Risk Management

\section{Publish your work in this journal}

Therapeutics and Clinical Risk Management is an international, peerreviewed journal of clinical therapeutics and risk management, focusing on concise rapid reporting of clinical studies in all therapeutic areas, outcomes, safety, and programs for the effective, safe, and sustained use of medicines. This journal is indexed on PubMed Central, CAS,
EMBase, Scopus and the Elsevier Bibliographic databases. The manuscript management system is completely online and includes a very quick and fair peer-review system, which is all easy to use. Visit http://www.dovepress.com/testimonials.php to read real quotes from published authors. 